\title{
Patient Perspectives on the Value of Patient Preference Information in Regulatory Decision Making: A Qualitative Study in Swedish Patients with Rheumatoid Arthritis
}

\author{
Karin Schölin Bywall ${ }^{1}$ ] . Jorien Veldwijk ${ }^{1,2}$. Mats G. Hansson ${ }^{1}$. Ulrik Kihlbom ${ }^{1}$
}

Published online: 15 November 2018

(c) The Author(s) 2018

\begin{abstract}
Background There is increasing interest in involving patient preferences for benefits and risks in regulatory decision making. Therefore, it is essential to identify patient perspectives regarding the value of patient preference information (PPI). Objectives The aim of this study was to explore how patients with rheumatoid arthritis (RA) value the use of PPI in regulatory decision making regarding medical products.

Methods Regulators and patients with RA were interviewed to gather initial insights into opinions on the use of PPI in regulatory decisions regarding medical products. The interviews were used to draft and validate the interview guide for focus groups with patients with RA. Participants were purposively sampled in collaboration with the Swedish Rheumatism Association in Stockholm and Uppsala. Each focus group consisted of three to six patients (18 in total). All interviews were audio-recorded, transcribed verbatim, and analysed using content analysis.

Results According to the participants, PPI could lead to regulators considering patients' needs, lifestyles and well-being when making decisions. PPI was important in all stages of the medical product lifecycle. Participants reported that, when participating in a preference study, it is important to be well-informed about the use of the study and the development, components, administration, and risks related to the medical products.

Conclusions Patients thought PPI could be valuable to consider in regulatory decisions. It is essential for patients to be well-informed when asked for their preferences. Research on information materials to inform patients in preference studies is needed to increase the value of PPI in regulatory decision making.
\end{abstract}

Electronic supplementary material The online version of this article (https://doi.org/10.1007/s40271-018-0344-2) contains supplementary material, which is available to authorized users.

Karin Schölin Bywall

karin.bywall@crb.uu.se

1 Centre for Research Ethics and Bioethics, Uppsala University, Husargatan 3, Box 564, 75237 Uppsala, Sweden

2 Erasmus School of Health Policy \& Management (ESHPM) and Erasmus Choice Modelling Centre (ECMC), Erasmus University Rotterdam, P.O. Box 1738, 3000 DR Rotterdam, The Netherlands

\section{Key Points for Decision Makers}

Patients thought it was important to be involved in regulatory decisions and that patient preference information (PPI) could be valuable to consider when including their preference.

PPI in regulatory decisions may ultimately lead to compliance with treatment, which could improve clinical outcomes in patients with rheumatoid arthritis.

Adequately informing patients about the medical product characteristics included in a preference study can increase the value of PPI in regulatory decision making. 


\section{Introduction}

Traditionally, regulatory decision making has attempted to balance the risks and benefits of medical products according to the outcomes of clinical trials. However, as regulators have come to recognise that they may not assign the same value to risks and benefits as would patients, they have begun to emphasise patients' perspectives [1]. Moreover, patients' perspectives regarding risks, benefits, and treatment procedures vary $[2,3]$. For example, not all patients would accept higher risks to gain a small benefit [4]. Patient perspectives are occasionally included in regulatory decision making via the inclusion of patient representatives in decision boards and groups in the EU [3]. Different instruments have also been developed to elicit and include patients' experiences, such as patient-reported outcomes, which measure clinically meaningful endpoints. However, these methods cannot capture the relative importance of different clinical endpoints or how patients make trade-offs between risks and benefits [5].

The increasing interest in involving patients' perspectives on risks and benefits has inspired the development of new methods that elicit and measure preferences in patient populations $[1,6]$. To quantify the relative importance of medical product characteristics, methods such as discretechoice experiments (DCEs) ask patients to choose treatment attributes [7]. In addition, patient perspectives can be assessed using patient preference information (PPI). According to the US FDA, PPI is "qualitative or quantitative assessments of the relative desirability or acceptability to patients of specified alternatives or choices among outcomes or other attributes that differ among alternative health interventions" [2]. PPI has been reported to support risk-benefit decisions by transparently framing issues from the patient's perspective $[8,9]$ and by identifying subgroups when preferences differ [10].

In both the EU [1] and the USA [6], regulatory agencies have acknowledged a need for more research that investigates how patient preferences are assessed. Moreover, agencies have not established best practices (i.e., the PPI methods to use in regulatory decision making), when to use PPI, or how best to conduct patient-preference studies. Although regulatory agencies recognize the value of PPI, few studies have evaluated how patients view the use of PPI for regulatory decision making. Patients' perspectives on PPI can provide input that can be used when recommending how to measure and implement PPI in regulatory decisions for drugs and devices in the medical product lifecycle (MPLC) $[5,9,11]$.

This article focuses on the perspectives of patients with rheumatoid arthritis (RA) on the value of including PPI in regulatory decisions regarding (new) disease-modifying antirheumatic drugs (DMARDs). RA-a chronic inflammatory autoimmune disease-is characterised by tenderness, swelling, and destruction of joints, which can result in disability and premature death [12]. The individual burden includes decline in physical function, causing musculoskeletal deficits and reduced work capacity, resulting in high socioeconomic burden. Early treatment with DMARDs can control and prevent negative outcomes such as pain, stiffness, fatigue, fever, weakness, deformity, malaise, weight loss, and depression [13, 14]. Because patients with RA are familiar with the disease, treatments, and side effects, their experiences can contribute to understanding how best to use PPI in regulatory decision making. The aim of this study was to explore how patients with RA value the use of PPI in regulatory decision making regarding medical products.

\section{Methods}

A two-step approach was used to explore how patients with RA value the use of PPI in regulatory decision making for medical products. First, semi-structured interviews with regulators and patients were conducted to develop an interview guide. Second, focus groups were conducted with patients with RA in Stockholm and Uppsala, Sweden. The study was approved by the regional ethics review board in Uppsala, Sweden (Reg. no. 2017/001). All participants in the interviews and focus groups provided written informed consent.

\subsection{Semi-Structured Interviews with Regulators and Patients}

Initially, four regulators from the Swedish Medical Products Agency were individually interviewed to map the current involvement of patients' preferences and the potential use of PPI in regulatory decision-making processes. An interview guide was developed in collaboration with the PREFER (Patient Preferences in Benefit-Risk Assessments in the Drug Lifecycle) project [15]. A selection of questions from the interview guide were used in this study because we focused on the regulatory process. The interviewees came from a pool of regulators in Sweden working with risk and benefit decisions at the national or EU level (i.e., at the Medical Products Agency and the European Medicines Agency). An invitation was emailed to these regulators, and all interviews were conducted face-to-face with the first author (KSB).

In addition, five patients with RA were interviewed to gather preliminary insights into patients' knowledge and opinions on the use of PPI in regulatory decision making. These interviews used another interview guide developed in the PREFER project. The guide was developed for patients, 
caregivers, and patient representatives and included questions about the participants' views on conducting and using the results from patient-preference studies in the MPLC. Patients in this study were purposively selected using the following inclusion criteria: aged $18-80$ years, established RA diagnosis reflecting one of the stages of treatment, and representing an RA patient organisation. The following patients were selected for interviews: one patient representing a patient organisation, one patient who was newly diagnosed (i.e., within 6 months), one patient treated with at least one synthetic DMARD, one patient receiving at least one biological DMARD, and one patient treated with synthetic and biological DMARDs. These patients were asked to participate in the interviews by their rheumatologist at Uppsala University Hospital. Invitations were sent via email to patients who volunteered to participate. The interviews were conducted by the first author (KSB) at Uppsala University or at the participants' workplace.

\subsubsection{Analysis of Semi-Structured Interviews}

The first author (KSB) conducted, audio-recorded, and transcribed the interviews, which were then analysed using content analysis [16]. Coding and analysis of the interviews were mainly performed by the first author (KSB) and the last author (UK), and results from both authors were compared and combined in discussion with all of the authors. All authors reached consensus on the codes, sub-categories, and main categories. This consensus generated topics for further exploration in focus groups with patients with RA. The analysis of the regulatory interviews revealed several themes: patient involvement in the regulatory process, the value of patient preferences in the regulatory process, patient preferences in the MPLC, methods for measuring patient preferences, and the need to inform patients. The regulatory interviews were also used to draft the educational material for the focus groups. Analysis of the patient interviews revealed the following themes: patient preferences in the regulatory process, patient preferences in the MPLC, methods for measuring patient preferences, and the need to inform patients. Results from the patient interviews and from the regulatory interviews were reviewed by all of the researchers. All authors reached consensus on three topics and questions for the interview guide for focus groups with patients with RA (Table 1).

\subsection{Focus Groups}

Four focus groups with patients with RA were conducted to explore the perspectives of patients on the value of PPI in regulatory decisions. 


\subsubsection{Recruitment}

Patients were purposively sampled from the Swedish Rheumatism Association in Stockholm and Uppsala. To ensure a mix of patients, the following inclusion criteria were used: aged 18-80 years, established RA diagnosis, and different stages of treatment. The Swedish Rheumatism Association in Stockholm and Uppsala sent invitations to patients. Each focus group consisted of three to six patients (18 in total).

\subsubsection{Conducting of Focus Groups}

Results from the semi-structured interviews were used to draft the interview guide for the focus groups. The guide was pre-tested using a pilot focus group $(n=4)$ and refined before the final focus groups were conducted. The interview guide consisted of the following topics: perceived value of RA patient preferences in the regulatory process, operationalisation of preference studies, and perceived need among patients to be informed. The focus groups were conducted by the first author (KSB) in Swedish, and an assistant took notes. The last author participated as an assistant in two of the focus groups. Before ending the focus group, the assistant summarised the discussion and gave the participants an opportunity to clarify what they said. The Swedish Rheumatism Association in Stockholm and Uppsala provided their facilities for two focus groups each. The focus groups lasted $\sim 120 \mathrm{~min}$, including a 20 -min presentation of the regulatory process and the potential to include PPI. The information provided in the presentation was identified using the semi-structured interviews.

\subsubsection{Data Analysis}

All focus groups were audio-recorded and transcribed verbatim. Data collection and analyses were performed concurrently by starting the analysis right after the first interview to estimate when thematic saturation was reached. A qualitative content analysis with a manifest and inductive approach was applied to analyse the focus groups [16]. In a first step, the transcripts were independently coded by two researchers (KSB, UK) to develop an initial list of codes. In this step, relevant fragments of text were extracted and coded. Similar codes that belonged to the same content were then grouped into potential sub-categories and main categories. Second, investigator triangulation was obtained in further analysis by discussing all potential sub-categories and main categories with all authors to further refine the list of codes. When all authors reached consensus, the refined list of codes was entered into the program NVivo 11 together with transcripts of the focus groups. The list of codes included 40 codes, 11 sub-categories, and three main categories (Table 2). After the content from the transcripts was coded, it was entered into NVivo. When the data were structured according to the list of codes, the authors (KSB, UK) independently summarised the quotations. These summaries were discussed among all of the authors and revised into a result. Meaningful quotations were selected from the data and translated into English to illustrate the relevance of the result.

\section{Results}

Most participants were highly educated females aged 28-79 years (mean 59). The duration since RA diagnosis was $1-42$ years (Table 3 ).

\subsection{Value of Using Rheumatoid Arthritis Patient Preference Information (PPI) in Regulatory Decisions}

Participants in the focus groups discussed the fact that patients should have the right to influence regulatory decisions:

"I actually think that you should claim the right to an opinion."

In addition, they discussed that studies that elicit patient preferences can provide them the opportunity to express their opinion. Several participants said it was important for regulators to consider patients' preferences when making decisions because patients may have varying preferences about what risks and benefits they want to consider:

"It might be the case that patients explain that they are willing to do different things. I mean that they value this differently, that it's okay to have some side effects as long as the benefit is greater, but other side effects may not be okay at all in the long run. I don't know. I think that decision makers might have a different view of benefits and risks than patients. So, it might be good for them to be given a voice there."

Participants also said that patient-preference studies could provide information to regulators about valuable characteristics for treatment from a great number of patients:

"The more patients influencing these decisions, the better, and if we say that this doesn't work, they need to do something about it. Well, I think that before experts make up their minds, they should get information from patients."

Currently, available treatments do not fit all participants' needs, lifestyles, and well-being. Some participants shared their experiences of unbearable side effects that made them stop their treatments: 
Table 2 List of codes

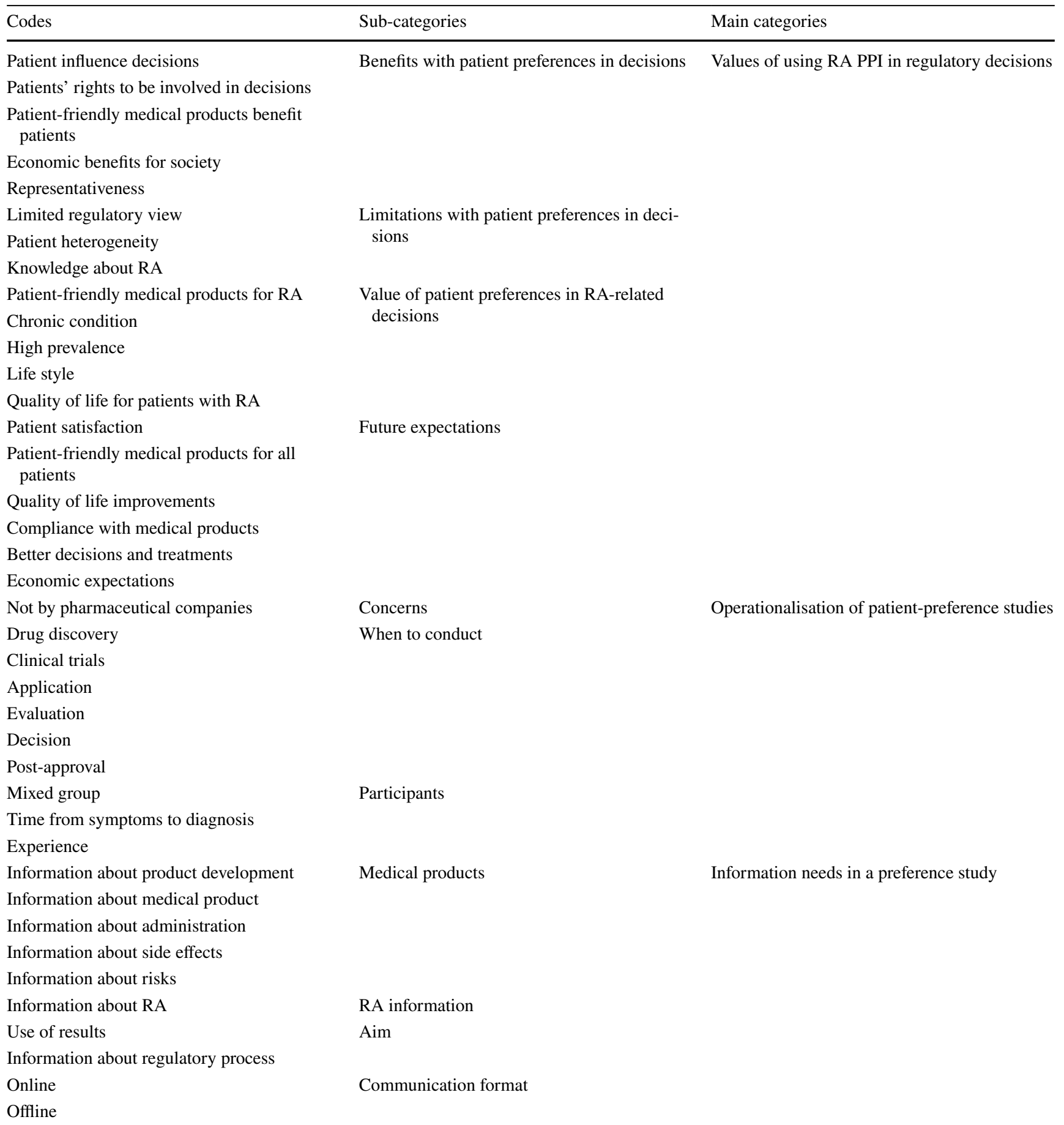

$P P I$ patient preference information, $R A$ rheumatoid arthritis

"From everything I went through, they completely failed me on this, you might say. They did for me, in this case. And I talked to my doctor and it wasn't just me, it was plenty of people. It was the same thing. So, that went totally wrong, and it makes you wonder." 
Table 3 Patients in focus groups $(N=18)$

\begin{tabular}{ll}
\hline Characteristic & $N^{\mathrm{a}}$ \\
\hline Sex & \\
Female & 17 \\
Male & 1 \\
Education level & 5 \\
Low & 5 \\
Medium & 8 \\
High & $59(28-79)$ \\
Mean age (range), years & $1-42$ \\
Years with RA (range) & \\
Interview location & 11 \\
Stockholm & 7 \\
Uppsala & \\
\hline
\end{tabular}

${ }^{\mathrm{a}}$ Unless otherwise indicated

Participants also mentioned some limitations with PPI, such as the limited knowledge patients have regarding medical products. According to some participants, this can result in an incorrect view among regulators of what patients prefer:

"I' $\mathrm{m}$ a bit hesitant about this, since my perspective is that we all have different medications, so what are we going to tell them then?"

Another limitation related to the ways in which patients with RA differ: disease trajectories, symptoms, and treatments, response to treatments, and side effects experienced:

"That one medicine doesn't work on the other person and the disease trajectory isn't the same for everybody."

Several participants stated that patients might have different preferences:

"Because people are very different, and the most important preferences are of course that the treatment can help, and that they have an effect, and that there are as few side effects as possible. The decision makers should also be aware that people are different."

Many participants said it was important to include PPI specifically in regulatory decisions regarding RA as RA is a chronic condition with a high prevalence:

"I would say that, maybe, it is extra important for RA because it is a chronic condition that has no cure."

Some participants stated that regulators should use PPI in decisions because patients with RA have more knowledge than regulators about their needs, lifestyles, and well-being:
"We are the ones with this disease; we know a lot about it and how it makes us feel. I think, because of that, they should take our opinions into consideration more often."

Furthermore, some participants said PPI might contribute to improved product efficacy and usability with respect to patients' lifestyles:

"Maybe more thorough decisions, so to speak, that you $[\ldots]$ yes $[\ldots]$ if they consider this for a longer time. So, this is the patient's preference and we also need to take this into consideration."

That is, medical products based on patient feedback might improve patient compliance with treatment. In addition, participants said that compliance might make it more likely that they would return to their daily lives and work, further easing the socioeconomic burden associated with RA:

"Well, these past 9 months, with so many things, I mean, I was going to [...] I was just lying there, just like in a coma from this medication. I couldn't work or do anything, which was just silly, I mean, that wasn't really saving money."

\subsection{Operationalisation of PPI}

Participants had different ideas on where in the MPLC it is most important to conduct patient-preference studies in order for PPI to be incorporated in regulatory decisions. When it comes to designing patient-friendly products, some participants said PPI is most important for drug discovery, but other participants said PPI is most important for clinical trials or the application phase:

"Possibly in clinical trials, if there's a possibility to provide something that a lot of people want, but they don't know."

Most participants discussed that PPI should be incorporated into risk-benefit evaluations because regulators make objective decisions on whether to approve a product:

"Absolutely before the decision so the experts conducting evaluations know what patients prefer."

Although participants said that PPI can benefit the development of patient-friendly products, they expressed concerns regarding pharmaceutical companies conducting studies. That is, some believed that pharmaceutical companies might care only about profits and "lie to patients":

"Yes, I thought so, it's both for and against, but I don't really trust the pharmaceutical companies, because it appears that they lie sometimes, so that's what I'm thinking about." 
Another participant expressed similar pessimism:

"I don't think the first steps are relevant because they are linked to the pharmaceutical company."

All of the groups discussed the importance of including a mixed group of patients regarding sex, age, socioeconomic position, and lifestyle in the preference studies:

"Yes, they should absolutely be of different ages and sexes and [...] yes, different occupations and different life situations, and how long you've had the disease and things like that. It's really important that it's not a homogenous group."

Many participants emphasised that patient-preference studies need to include patients with experience of the disease and of treatments:

"Yes, then you can't have recently diagnosed patients; it won't work. Experience with the disease and offering valuable insights requires you to have had it for some time and to have tried different things."

\subsection{Information Needs in a Preference Study}

The participants discussed that patients need to be informed about how the results will be used and where in the regulatory process a study will have an influence:

"I would like to know if it's before a decision, or if it's already decided; then it is what it is, then I might not be able to have an influence the same way as before the decision."

They also said patients should be given information about RA and the medical products-e.g., their development, components, administration, and risks-before participating in a preference study:

"I would like to know how this medical product acts in the body. What does it do and what is happening, what are the possible side effects?"

Most of the participants said they prefer written information and questionnaires over interviews because it gives them time to go back and think about their answers:

"Because then you can go back and forward and think about it; you can return to the questions another day."

Online surveys might be suitable, according to some participants, since they allow for more flexible interactivity with the questionnaire. They also said that online surveys might be more convenient for patients with RA to answer, because it is easier for them to press buttons than to write with a pen:

"With our disease especially, it's easier to press a button than to write."

\section{Discussion}

The use of PPI in regulatory decision making regarding medical products has been emphasised in several initiatives to investigate methods that elicit patient preferences for regulatory decisions $[1,6]$. This study brings a new aspect to this perspective by asking patients about their view on PPI in regulatory decision making.

The main finding in this study was that patients think PPI could provide valuable information for regulatory decision making. According to the participants, PPI could benefit both the patients and the regulators in that the regulators could take patients' needs, lifestyles, and well-being into consideration when evaluating and approving (including post-approval) medical products. Both stakeholders could also benefit from PPI through the ability to identify and value important treatment characteristics for patients, as regulators may not be adequately informed about patient perspectives when only referring to the results of clinical trials. For example, PPI can provide information about the relative importance of different medical product characteristics. This finding is consistent with previous findings that PPI is important to consider in regulatory decisions [10,17].

The participants also discussed possible limitations with PPI, such as an inability to capture how patients differ in their preferences because the preferences of patients with RA do vary [18]. However, preference studies can make it possible to present how preferences differ within subgroups of the patient population. Methods that elicit patient preferences, such as DCEs, can also quantify the relative importance of risks and benefits of medical products and make the information valuable for regulatory decisions [10].

In addition, participants expressed that PPI should be used to complement information from clinical trials. This finding is in line with those of previous research, that regulators should consider how patients weigh risks and benefits when making regulatory decisions [1]. Participants also recognise that the risk and benefit evaluation is an important step in the MPLC for implementing the use of PPI. Patients may adopt this reasoning because they do not want market approval for medical products that could expose them to a certain side effect [4].

Some participants expressed concern about pharmaceutical companies conducting patient-preference studies, with some thinking pharmaceutical companies should not be involved in conducting preference studies. One reason for expressing this concern might be a worry that pharmaceutical companies introduce bias to PPI. Other participants thought it would be good for pharmaceutical companies to have the information early in the product-development process. 
A third finding was the identified need to inform participants in preference studies about the use of the study, the disease, and the medical product's characteristics. Participants may have expressed a need for information because it was important to them that patient preferences used in regulatory decision making are representative and well-informed. This need for information may also relate to their desire that participants in preference studies represent a range of experiences with the disease and its treatments. However, adequately informing patients about the medical product characteristics included in the preference study might decrease this concern by increasing the participants' ability to make a well-informed choice when answering the questions.

This study has several limitations. Patients in the focus groups were approached by their patient organisation, which could influence their perspective toward including PPI in regulatory decision making. Another possible limitation is selection bias, since the patient sample mostly consisted of highly educated females aged $>50$ years. Although this was partly expected because the prevalence of RA increases with age and is about twice as high in women as in men [19], it might have slightly influenced study outcomes.

\section{Conclusions}

Patients think it is important to be involved in regulatory decisions regarding medical products and believe that PPI could be a valuable tool for assessing the preferences of patients. When answering a preference study, it is essential that patients are well-informed about the use of preference data, the disease, and the medical product characteristics. Further research that focuses on methods for informing participants in a preference-elicitation study is needed to increase the value of PPI in regulatory decision making.

Acknowledgements The authors thank the Swedish Rheumatism Association for their support in the recruitment of participants and offering their agency for conducting the focus groups. We would also like to thank the members in the PREFER project involved in developing the interview guide for the semi-structured interviews, especially Isabelle Huys, Rosanne Janssens, Eline van Overbeeke, Chiara Whichello, and Selena Russo.

Data availability The datasets generated during and/or analysed during the current study are available from the corresponding author upon request.

Author contributions The interview guides for the semi-structured interviews were developed in collaboration with the project 'Patient Preferences in Benefit-Risk Assessments in the Drug Lifecycle' (PREFER). KSB, JV, MGH, and UK designed the interview guide for the focus groups. All interviews were conducted by KSB. KSB and UK were responsible for the analysis and drafted the manuscript. All authors reviewed the final version and approved it for publication.

\section{Compliance with Ethical Standards}

Funding This work was supported by The Swedish Foundation for Humanities and Social Sciences (Riksbankens Jubileumsfond) [grant number M13-0260:1].

Conflicts of interest Karin Schölin Bywall, Jorien Veldwijk, Mats G. Hansson, and Ulrik Kihlbom have no conflicts of interest that are directly relevant to the content of this article.

Ethical approval This study was approved by the regional ethics review board in Uppsala, Sweden (Reg. no. 2017/001). All participants in the interviews and focus groups provided written informed consent. All procedures performed in studies involving human participants were in accordance with the ethical standards of the institutional and/or national research committee and with the 1964 Helsinki declaration and its later amendments or comparable ethical standards. Informed consent was obtained from all individual participants included in the study.

Open Access This article is distributed under the terms of the Creative Commons Attribution-NonCommercial 4.0 International License (http://creativecommons.org/licenses/by-nc/4.0/), which permits any noncommercial use, distribution, and reproduction in any medium, provided you give appropriate credit to the original author(s) and the source, provide a link to the Creative Commons license, and indicate if changes were made.

\section{References}

1. Mühlbacher AC, Juhnke C, Beyer AR, Garner S. Patient-focused benefit-risk analysis to inform regulatory decisions: the European Union Perspective. Value Health. 2016;19(6):734-40.

2. Patient Preference Information-Voluntary Submission, Review in Premarket Approval Applications, Humanitarian Device Exemption Applications, and De Novo Requests, and Inclusion in Decision Summaries and Device Labeling: Guidance for Industry, Food and Drug Administration Staff, and Other Stakeholders. U.S. Department of Health and Human Services, Food and Drug Administration, Center for Devices and Radiological Health and Center for Biologics Evaluation and Research. 2016.

3. Regulatory and methodological standards to improve benefitrisk evaluation of medicines. European Medicines Agency, Stakeholders and Communication Division2014. Report No.: EMA/141854/2014

4. Hazlewood GS, Bombardier C, Tomlinson G, Thorne C, Bykerk VP, Thompson A, et al. Treatment preferences of patients with early rheumatoid arthritis: a discrete-choice experiment. Rheumatology (Oxford, England). 2016;55(11):1959-68. https://doi. org/10.1093/rheumatology/kew280.

5. van Til JA, Ijzerman MJ. Why should regulators consider using patient preferences in benefit-risk assessment? Pharmacoeconomics. 2014;32(1):1-4. https://doi.org/10.1007/s40273-013-0118-6.

6. Johnson FR, Zhou M. Patient preferences in regulatory benefit-risk assessments: a US perspective. Value Health. 2016;19(6):741-5.

7. Lancsar E, Louviere J, Flynn T. Several methods to investigate relative attribute impact in stated preference experiments. Social science \& medicine (1982). Soc Sci Med. 2007;64(8):1738-53. https://doi.org/10.1016/j.socscimed.2006.12.007.

8. Egbrink MO, M IJ. The value of quantitative patient preferences in regulatory benefit-risk assessment. Journal of market access \& health policy. 2014. https://doi.org/10.3402/jmahp.v2.22761. 
9. Ho M, Saha A, McCleary KK, Levitan B, Christopher S, Zandlo $\mathrm{K}$, et al. A Framework for incorporating patient preferences regarding benefits and risks into regulatory assessment of medical technologies. Value Health. 2016;19(6):746-50.

10. Ho MP, Gonzalez JM, Lerner HP, Neuland CY, Whang JM, McMurry-Heath M, et al. Incorporating patient-preference evidence into regulatory decision making. Surg Endosc Other Interv Tech. 2015;29(10):2984-93.

11. Morel T, Ayme S, Cassiman D, Simoens S, Morgan M, Vandebroek M. Quantifying benefit-risk preferences for new medicines in rare disease patients and caregivers. Orphanet J Rare Dis. 2016;11(1):70. https://doi.org/10.1186/s13023-016-0444-9.

12. Aletaha D, Neogi T, Silman AJ, Funovits J, Felson DT, Bingham CO 3rd, et al. 2010 Rheumatoid arthritis classification criteria: an American College of Rheumatology/European League Against Rheumatism collaborative initiative. Arthritis Rheum. 2010;62(9):2569-81. https://doi.org/10.1002/art.27584.

13. Smolen JS, Aletaha D, McInnes IB. Rheumatoid arthritis. Lancet (London, England). 2016;388(10055):2023-38. https://doi. org/10.1016/s0140-6736(16)30173-8.

14. Lee DM, Weinblatt ME. Rheumatoid arthritis. Lancet (London, England). 2001;358(9285):903-11. https://doi.org/10.1016/s0140 $-6736(01) 06075-5$.
15. de Bekker-Grob EW, Berlin C, Levitan B, Raza K, Christoforidi $\mathrm{K}$, Cleemput I, et al. Giving patients' preferences a voice in medical treatment life cycle: the PREFER public-private project. Patient. 2017;10(3):263-6. https://doi.org/10.1007/s4027 1-017-0222-3.

16. Elo S, Kyngas H. The qualitative content analysis process. J Adv Nurs. 2008;62(1):107-15. https://doi.org/10.111 $1 / \mathrm{j} .1365-2648.2007 .04569 . x$.

17. Johnson FR, Beusterien K, Ozdemir S, Wilson L. Giving patients a meaningful voice in united states regulatory decision making: the role for health preference research. Patient. 2017;10(4):523-6. https://doi.org/10.1007/s40271-017-0250-z.

18. Fraenkel L, Nowell WB, Michel G, Wiedmeyer C. Preference phenotypes to facilitate shared decision-making in rheumatoid arthritis. Ann Rheum Dis. 2017. https://doi.org/10.1136/annrh eumdis-2017-212407.

19. Scott DL, Wolfe F, Huizinga TW. Rheumatoid arthritis. Lancet (London, England). 2010;376(9746):1094-108. https://doi. org/10.1016/s0140-6736(10)60826-4. 